\title{
CMOS COMPATIBILITY OF A MICROMACHINING PROCESS DEVELOPED FOR SEMICONDUCTOR NEURAL PROBE
}

\author{
S. K. An, S. J. Oh and S. J. Kim \\ School of Electrical Engineering and Nano Bioelectronics and Systems ERC, Seoul National University, Korea
}

\begin{abstract}
Neural probes are made on silicon substrate using a micromachining process with low temperature steps only. A deep silicon etch ("Bosch") process was used for the probe shaping. CMOS compatibility of the process was checked and reported in this paper. Test transistor patterns generated using standard CMOS fabrication line were exposed to a post-CMOS probe making process including dielectric deposition, gold metalization and the dry etching step, while changes of test transistor characteristics were monitored. Threshold voltage was found virtually unchanged for both nand p-type MOS transistors. When excess plasma exposure was done, however, non-trivial shift in p-MOS threshold was observed.
\end{abstract}

Keywords - Neural probes, CMOS compatibility

\section{INTRODUCTION}

Silicon-based microelectrode for the neural recording and stimulating have been developed because the silicon microelectrode have many advantages over conventional metal wire bundle[1]. Active circuitry on the silicon microelectrode can be monolithically integrated so that impedance buffering, amplification, and multiplexing can be done[2]. We have previously reported on the process of making a new type of silicon microelectrode[3]. The shank of the microelectrode was made controllable with use of dry etch on the front side and backside. In contrast to the process developed in University of Michigan, our process uses only low temperature steps so it can be applied to completed CMOS chips. The advantage of this approach is that we can use the vendor CMOS services such as MOSIS. This paper reports on the methods and results of the experiments we performed to verify the CMOS compatibility of the probe shaping steps we developed.

\section{METHODOLOGY}

\section{A. Probe Shaping Process}

A needle-like shank of a neural probe was fabricated by isotropic etching of the backside of a silicon wafer after making ridge-like structures on front-side. A deep silicon etch process - called Bosch process - was used. The etch rate was about $2 \mu \mathrm{m} / \mathrm{min}$ and the etch selectivity against positive photo-resist was more than 30 . When a photoresist of $5 \mu \mathrm{m}$ thickness is used, we can easily control the thickness of probe-shank up to $150 \mu \mathrm{m}$.

\section{B. Test Circuit Design}

MOSFET patterns and source-followers were designed in order to specify the CMOS compatibility with the micromachining post-process. We varied the gate lengths of n-type MOSFETs and p-type MOSFETs to be $1.5 \mu \mathrm{m}, 2 \mu \mathrm{m}, 3$ $\mu \mathrm{m}$, and $5 \mu \mathrm{m}$, with fixed gate widths of $20 \mu \mathrm{m}$. In addition to the test MOSFET patterns, source-follower that was widely used as a unity gain buffer of typical neurophysiological experiment setup was also integrated. P-type MOSFETs were used because they, with large gate dimensions, are known to have reduced noise levels when used in sourcefollowers[4].

\section{Fabrication}

The test transistors and circuits were fabricated using a 1.5 um standard CMOS process service offered by the ISRC(Interuniversity Semiconductor Research Center) of 


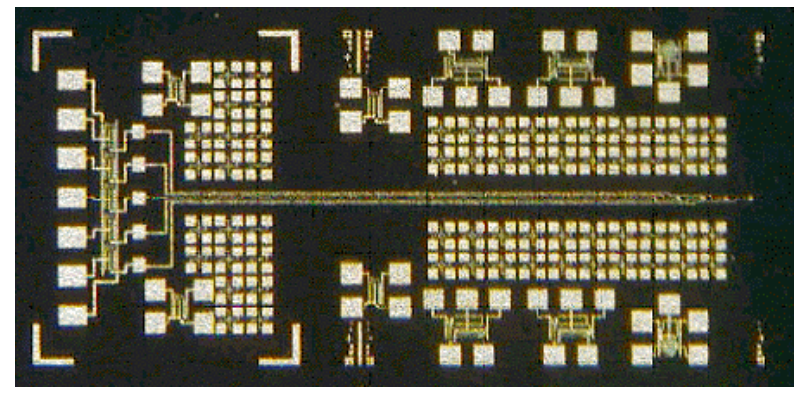

Fig.1, Photomicrograph of a completed circuit die. The chip is fabricated using a $1.5 \mu \mathrm{m}$ CMOS process with a die size of $3 \mathrm{~mm} \times 6 \mathrm{~mm}$.

the SNU(Seoul National University). This process is a double metal, single poly process with a gate oxide thickness of $250 \AA$. After the CMOS process, the micromachining process discussed above was performed to achieve the structure of the neural probe. Figure 2 shows the process flow. First, a triple dielectric layer consisting of PECVD silicon dioxide(200nm), silicon nitride(200nm), and silicon dioxide $(800 \mathrm{~nm})$ was deposited on the wafer completed with the CMOS process. The recording sites were then opened by plasma etch and inlaid with gold. Bonding pads were also opened as shown in figure 2(b). The front side of a wafer was then etched to make the ridgelike structures as shown in figure 2(c). This Bosch process was done at $9 \mathrm{~W}$ power for 45 minutes. Finally the backside of the wafer was etched out using isotropic etching and the process was completed.

\section{Measurement}

In order to verify the CMOS compatibility of the postCMOS micromachining process, the threshold voltages of test MOS patterns and the DC characteristics of sourcefollowers were measured at the following three phases: 1) after the standard CMOS process, 2) after the site opening and gold metalization, and 3) after the deep silicon etching. A probe station and a semiconductor parameter analyzer (4155A, Hewlett Packard) were used in circuit tests.

\section{RESULTS}


the CMOS compatibility of the said process, we designed MOS patterns and a source-follower, and tracked their characteristics at three phases of the process. We could verify the CMOS compatibility of the process and proved that the low power Bosch etch process did not cause significant plasma damage that causes threshold changes. However, when a plasma etch process at higher power and for longer duration was added, some shifts in thresholds (a shift of a few tenths of a volt) occurred in the case of p-type MOS. The reason why it affected $\mathrm{p}$-type MOS but not the $\mathrm{n}$ type one, requires further investigation. Furthermore, such high power, MOS-damaging plasma etch process for the backside would not be required if the backside etch is done employing one of the widely used wet chemical etching techniques, such as EDP and $\mathrm{KOH}$ etching.

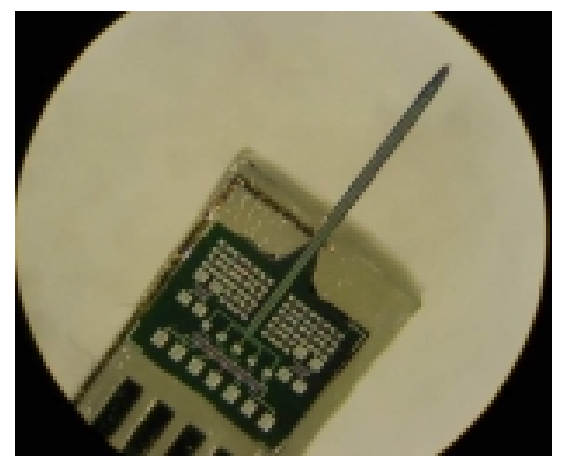

Fig.3, Photograph of a silicon microelectrode with on-chip test MOSFET patterns and source-followers. The overall length of the probe is $6 \mathrm{~mm}$.

(a) Gate Length vs Threshold Voltage (PMOS)

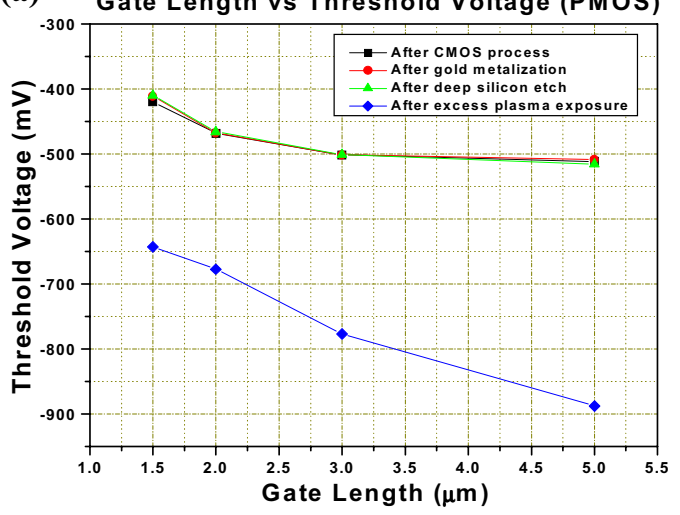

(b)

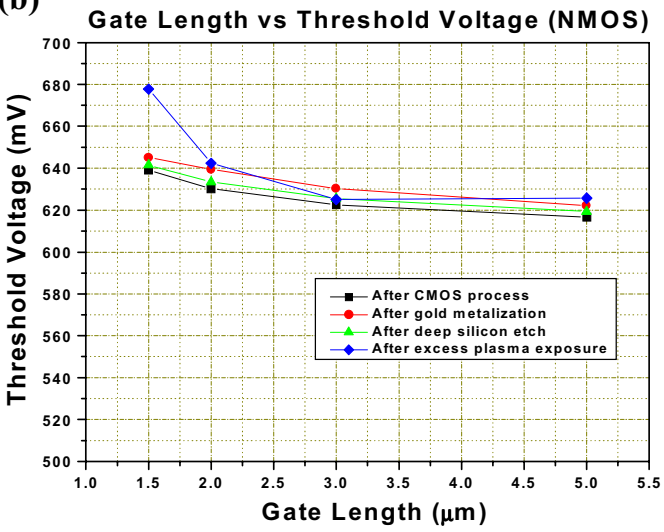

(c) Source follower DC characteristic curve

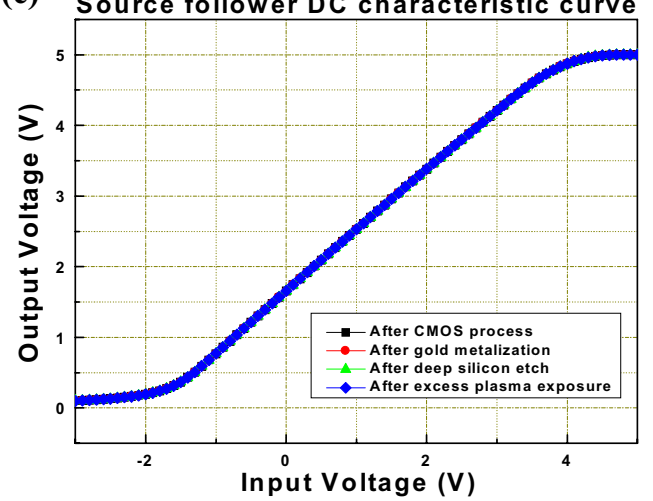

Fig.4, The changes of DC characteristics during the probe-shaping micromachining process of (a) PMOSFETs, (b) NMOSFETs, and (c) source followers.

\section{REFERENCES}

[1] Arnold Starr, Kensall D. Wise and John Csongradi, "An Evaluation of Photoengraved Microelectrodes for Extracellular Single-Unit Recording," IEEE Transactions on Biomedical Engineering, vol. 20, pp. 291-293, July 1973.

[2] Khalil Najafi, Kensall D. Wise, “An Implantable Microelectrode Array with On-Chip Signal Processing," IEEE Journal of solid-state circuits, vol. sc-21, no. 6, pp. 1035-1044, December 1986.

[3] T.H.Yoon,E.J.Hwang,D.Y.Shin,S.I.Park,S.J.Oh,S.C.Jung,H.C.Shin, and S.J.Kim, "A Micromachined Silicon Depth Probe for Multi-Channel Neural Recording," IEEE Transactions on Biomedical Engineering, vol. 47, pp. 1082-1087, August 2000.

[4] K.H.Kim and S.J.Kim, "Noise characteristics design of CMOS source follower and voltage amplifier for the active semiconductor microelectrode for neural signal recording", Medical and Biological Engineering and 
Computing, vol. 38, pp. 469-472, July 2000.

[5] K. Najafi and J. Hetke, "Strength characterization of silicon microprobes in neurophysiological tissues," IEEE Transactions on

Biomedical Engineering, vol. 37, pp. 474-481, May 1990. 